\title{
Instilación intratraqueal de elastasa en la rata: Cambios electroforéticos de la $\alpha 1$-antitripsina en el lavado broncoalveolar
}

\author{
ANDREA VECCHIOLA C.*, ALEJANDRO RAMÍREZ M.*, ANDREA VILLAGRÁN T.*, \\ CAROLINA SERRANO H.*, LEONEL LIBERONA T.*, CLAUDIA SÁEZ S.* y GISELLA BORZONE T.*
}

\begin{abstract}
Intratracheal instillation of elastase in the rat: Electrophoretical changes of alpha1-antitrypsin in bronchoalveolar lavage fluid
\end{abstract}

Introduction: Endogenous alpha1-antitrypsin $(\alpha 1-A T)$ is the main inhibitor of the intratracheally instilled elastase in experimental animals. Objective: To evaluate by electrophoresis and immunodetection using western blot analysis, the different forms of $\alpha 1$-AT in bronchoalveolar lavage fluid (BALF) of Sprague Dawley rats after intratracheal instillation of elastase, with the hypothesis that the previously observed increment in antielastase activity is due to high levels of active $\alpha 1-A T$. Results: In the first hours after elastase instillation the concentration of $\alpha 1-A T$ increases more than seven times due to an increase in alveolar-capillary permeability. $\alpha 1-A T$ in $B A L F$ is found as the native protein ( $52 \mathrm{kDa}$ ), as complexes of different molecular sizes (> $75 \mathrm{kDa}$ and $>100 \mathrm{kDa})$ and as a proteolytic product $(<40 \mathrm{kDa})$. Conclusion: In spite of a high proportion of $\alpha 1-A T$ in the inactive form as part of different complexes, the increase in alveolar-capillary permeability after elastase treatment contributes to maintain high levels of active $\alpha 1-A T$. These results could be of importance in other inflammatory lung processes.

Key words: antiprotease, $\alpha 1-A T$, diffuse lung damage, elastase, electrophoresis.

\section{Resumen}

Introducción: La antiproteasa alfa1-antitripsina ( $\alpha 1-A T)$ constituye el principal inhibidor endógeno de la elastasa instilada por vía intratraqueal en modelos experimentales. Objetivo: Evaluar mediante electroforesis e inmunodetección por western blot, las distintas formas en que se encuentra la $\alpha 1-A T$ en el lavado broncoalveolar (LBA) de ratas Sprague Dawley después de la instilación de elastasa, con la hipótesis de que el aumento en la actividad antielastasa previamente encontrada se acompaña de niveles altos de $\alpha 1$-AT activa. Resultados: En las primeras horas postelastasa la concentración de $\alpha 1-A T$ en el LBA aumenta más de 7 veces, debido al aumento de la permeabilidad alvéolo-capilar, encontrándose tanto como proteína nativa ( $52 \mathrm{kDa}$ ), como parte de complejos de mayor tamaño (> $75 \mathrm{kDa} \mathrm{y}>100 \mathrm{kDa}$ ) y como producto de proteólisis $(<40 \mathrm{kDa})$. Conclusión: A pesar de existir una alta proporción de $\alpha 1$-AT inactiva formando complejos, el aumento de la permeabilidad alvéolo-capilar contribuye a mantener niveles altos de $\alpha 1$-AT activa. Estos resultados podrían ser extrapolables a distintos procesos inflamatorios pulmonares.

Palabras clave: antiproteasa, $\alpha 1-A T$, daño pulmonar difuso, elastasa, electroforesis.

\section{Introducción}

La principal defensa contra las proteasas capaces de destruir la matriz extracelular del pul- món radica en la antiproteasa alfa1-antitripsina ( $\alpha 1-\mathrm{AT})$, una glicoproteína de $52 \mathrm{kDa}$, que si bien es capaz de inhibir a distintas proteasas, es altamente específica para la elastasa liberada des-

* Departamento de Enfermedades Respiratorias y Centro de Investigaciones Médicas, Pontificia Universidad Católica de Chile.

Financiamiento: Fondecyt:1030412, 11070121 
de los macrófagos y polimorfonucleares neutrófilos en distintos tipos de procesos inflamatorios pulmonares agudos y crónicos ${ }^{1-3}$. Más del $90 \%$ de la elastasa producida por estas células en el pulmón es inhibida por esta antiproteasa ${ }^{4}$. Dada su alta constante de asociación en su reacción con la elastasa, forma complejos estables en una "reacción suicida" que impide el daño elastolítico de los componentes de la matriz extracelular 5 .

Diversos estudios han mostrado que la deficiencia de $\alpha 1$-AT se acompaña de una inadecuada protección del tracto respiratorio inferior contra las proteasas, lo que favorece la destrucción de los tabiques alveolares ${ }^{6}$. En animales de experimentación por otra parte, la instilación intratraqueal (IT) de elastasa ha sido ampliamente utilizada para estudiar las consecuencias de un exceso de proteasas las que sobrepasan a la principal defensa antielastasa, favoreciendo la destrucción tisular?.

En un estudio previo, utilizando el modelo de instilación IT de elastasa en la rata Sprague Dawley, encontramos que la actividad de $\alpha 1$ AT en el lavado broncoalveolar (LBA) no disminuye como se esperaría debido a la formación de complejos con la elastasa instilada, sino que aumenta $^{8}$. Basado en lo anterior, nuestra hipótesis es que si bien la $\alpha 1-\mathrm{AT}$ existente en el pulmón es utilizada en la formación de complejos elastasa- $\alpha 1-A T$, existen mecanismos que favorecen la llegada de nueva $\alpha 1-\mathrm{AT}$ desde la circulación a los espacios aéreos, aumentando su concentración y actividad.

Nuestro objetivo fue estudiar en el LBA del pulmón de la rata a distintos tiempos después de la instilación IT de elastasa, la concentración de $\alpha 1-\mathrm{AT}$ y las formas en que ésta se encuentra, utilizando técnicas de electroforesis e inmunodetección. El conocimiento de la dinámica de los cambios experimentados por la $\alpha 1$-AT en el pulmón frente a una sobrecarga de proteasas contribuirá a una mejor comprensión de la fisiopatología de diversas enfermedades pulmonares en las cuales participa la liberación de proteasas.

\section{Método}

Modelo experimental de instilación de elastasa: El estudio se realizó de acuerdo a las normas del Comité de Ética para el trabajo con animales de la Pontificia Universidad Católica de Chile. Ratas Sprague-Dawley machos de $270 \pm$ $10 \mathrm{~g}$ de peso recibieron una dosis única de elastasa pancreática porcina (Sigma, 0,55 U/100 g de peso corporal en $0,5 \mathrm{ml}$ de $\mathrm{NaCl} 0,15 \mathrm{M}$ ) por vía IT. La solución de elastasa se preparó bajo condiciones estériles, inmediatamente antes de su administración IT con una aguja hipodérmica 25G bajo anestesia con hidrato de cloral (45 mg/100 g de peso corporal). Para ello se realizó una pequeña incisión en la piel del cuello para separar los músculos cervicales y exponer la tráquea. Luego del cierre de la incisión y de la recuperación anestésica, los animales fueron mantenidos en el vivero, con un régimen de luz de 12 horas diarias y alimentados ad libitum con alimento estándar para roedores. Se estudiaron cuatro grupos de animales (4 horas, 24 horas, 4 días, 7 días; $n=5$ por grupo, descontando una letalidad del 20\%). El grupo control $(n=5)$ estuvo constituido por animales sin intervención, mantenidos en similares condiciones que los grupos experimentales y anestesiados al momento del sacrificio.

Lavado Broncoalveolar: Inmediatamente después del sacrificio bajo anestesia con hidrato de cloral (45 mg/100 g de peso), los pulmones fueron resecados en bloque. El pulmón derecho fijado en formalina se utilizó para análisis histológico mientras que el pulmón izquierdo se utilizó para el LBA. Tres alícuotas de $2 \mathrm{ml}$ de solución salina $0,15 \mathrm{M}$, fueron instiladas, inmediatamente aspiradas, mezcladas y almacenadas a $-80{ }^{\circ} \mathrm{C}$ hasta su utilización en la determinación del contenido total de proteínas por el método de Bradford $^{9}$ y en la inmunodetección de $\alpha 1$ AT por western blot.

Electroforesis para la separación de las proteínas del lavado e inmunodetección de $\alpha 1$ AT: Con el objetivo de evidenciar las formas electroforéticas de $\alpha 1$-AT utilizamos geles de poli-acrilamida al 7,5\% tanto: a) en condiciones nativas (en ausencia de SDS y $\beta$-mercaptoetanol), lo que permite mantener la esctructura tridimensional de las proteínas que se intenta separar, como b) en condiciones desnaturantes que hacen desaparecer las estructuras terciarias y los complejos de $\alpha 1-A T$. Cada uno de los geles contenía cinco muestras de $20 \mu \mathrm{L}$ de LBA sin diluir (una muestra control y una muestra correspondiente a cada uno de los tiempos postelastasa) constituyendo un set. Concluida la electroforesis, las proteínas de algunos geles fueron teñidas con azul de Coomasie (Coomasie R250 en 10\%-ácido acético/45\%-metanol), mientras las restantes fueron transferidas a membranas de nitrocelulosa para la inmunodetección de $\alpha 1-\mathrm{AT}$. 
Inmunodetección de $\alpha 1-A T^{10}$. Luego de la separación de las proteínas mediante electroforesis, éstas fueron inmovilizadas en membranas de nitrocelulosa (PIERCE, tamaño de poro 0,45 $\mu \mathrm{m})$, las cuales fueron luego incubadas con una solución de gelatina 1\% en Tween-PBS previo a la reacción con el anticuerpo primario policlonal anti $\alpha 1-A T$ humana, (SIGMA, USA; con reacción cruzada para $\alpha 1-\mathrm{AT}$ de rata, dilución 1 : 1.000) y el anticuerpo secundario acoplado a peroxidasa de rabanito (PIERCE, Rockford, USA; dilución 1: 5.000). Se utilizó quimioluminiscencia (Amersham, Oakville, ON, Canadá) para revelar el perfil de las bandas obtenidas. La intensidad de las bandas se cuantificó mediante densitometría utilizando el programa Image $\mathrm{J}$ de procesamiento de imágenes desarrollado en The National Institutes of Health, USA.

Análisis estadístico: Los resultados fueron examinados utilizando análisis de varianza (ANOVA) y el test post-hoc de Tukey ${ }^{12}$. Un valor de $\mathrm{p}<$ de 0,05 fue considerado estadísticamente significativo.

\section{Resultados}

Evidencias de aumento de la permeabilidad alvéolo-capilar luego de la instilación de elastasa.

La Figura 1 muestra, en un gel representativo teñido con azul de Coomasie, el curso temporal de los cambios en la concentración de distintas proteínas en el lavado (LBA) después de la instilación de elastasa, en comparación con una muestra de suero (S) del mismo animal del cual se obtuvo el LBA. Es posible observar que en el grupo control la muestra de suero (S) y de lavado (LBA) difieren en el contenido y movilidad de las proteínas. A las cuatro horas después de la instilación IT de elastasa, el LBA presenta un cambio en el perfil de las proteínas, el que a las 24 horas es muy similar al del suero, como reflejo de un gran aumento de la permeabilidad alvéolo-capilar. Por otra parte, a los siete días, el perfil de las proteínas del LBA difiere del perfil del suero y es similar al del LBA basal.

En la Tabla 1 se muestra la concentración de proteínas totales en el LBA a los distintos tiempos, medida por el método de Bradford (en $\mu \mathrm{g}$ $\mathrm{x} \mathrm{ml} \mathrm{m}^{-1}$ ). Se puede inferir que el aumento de permeabilidad alvéolo-capilar es evidente, aunque no estadísticamente significativo, ya a las 4 horas post elastasa, dado el aumento de 4 veces en la concentración de proteínas totales en comparación con el grupo control. La concentración de proteínas en el LBA alcanza un máximo de 7 veces el valor del control a las 24 horas $(\mathrm{p}<0,001)$ y retorna a los niveles basales a los 4 y 7 días.

Aumento de la concentración de a1-AT en el LBA post-instilación de elastasa en relación al aumento de la permeabilidad alvéolo-capilar.

La Figura 2 ilustra la inmuno-reactividad en-

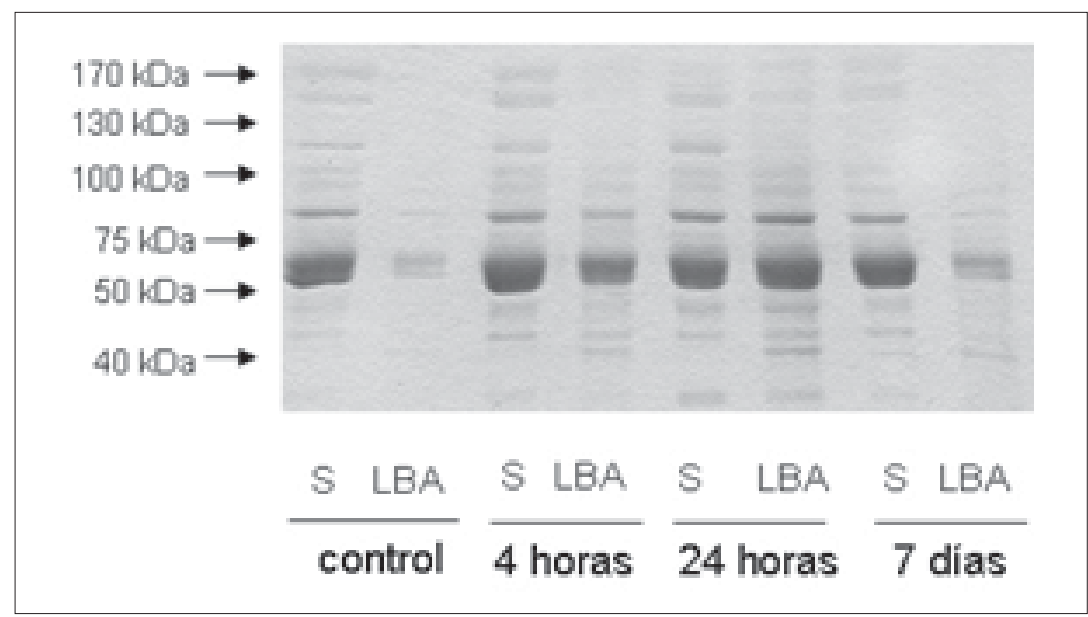

Figura 1. Curso temporal de los cambios en la concentración de distintas proteínas en el LBA después de la instilación de elastasa. Gel representativo teñido con azul de Coomasie para una muestra de suero (S) y otra de lavado broncoalveolar (LBA) proveniente de un mismo animal en condiciones basales y a distintos tiempos después de la instilación de elastasa. Las proteínas del LBA aumentan progresivamente hasta mostrar un perfil similar al del suero a las 24 horas, lo cual refleja el aumento en la permeabilidad alvéolo-capilar. A los siete días, el perfil de las proteínas del LBA es similar al del LBA control. 
contrada frente al anticuerpo anti $\alpha 1$-AT de las muestras y del LBA transferidas a una membrana de nitrocelulosa después de una electroforesis en condiciones desnaturantes. En A, el gráfico muestra los niveles de $\alpha 1$-AT inmuno-reactiva en el LBA obtenidos mediante análisis densitométrico a distintos tiempos después de la instila- ción de elastasa y expresados como veces de aumento en relación al control. En B se muestra un gel representativo, en el que es posible observar un aumento en la intensidad de la banda única de $52 \mathrm{kDa}$ correspondiente al peso molecular descrito para la $\alpha 1-A T$. Este aumento es evidente desde las 4 horas post-elastasa, y

Tabla 1. Contenido de proteínas totales en el LBA a distintos tiempos después de la instilación IT de elastasa

\begin{tabular}{lccccc} 
& Control & 4 horas & 24 horas & 4 días & $\mathbf{7}$ días \\
\hline $\begin{array}{l}\text { Proteínas }\left(\mu \mathrm{g} \mathrm{m} \mathrm{m}^{-1}\right) \\
(\text { Promedio } \pm \mathrm{DS})\end{array}$ & $5,2 \pm 6,8$ & $23,3 \pm 18$ & $38,8 \pm 28,4 * *$ & $2,9 \pm 2,5$ & $5,4 \pm 3,1$ \\
\hline
\end{tabular}

$* *: \mathrm{p}<0,01$

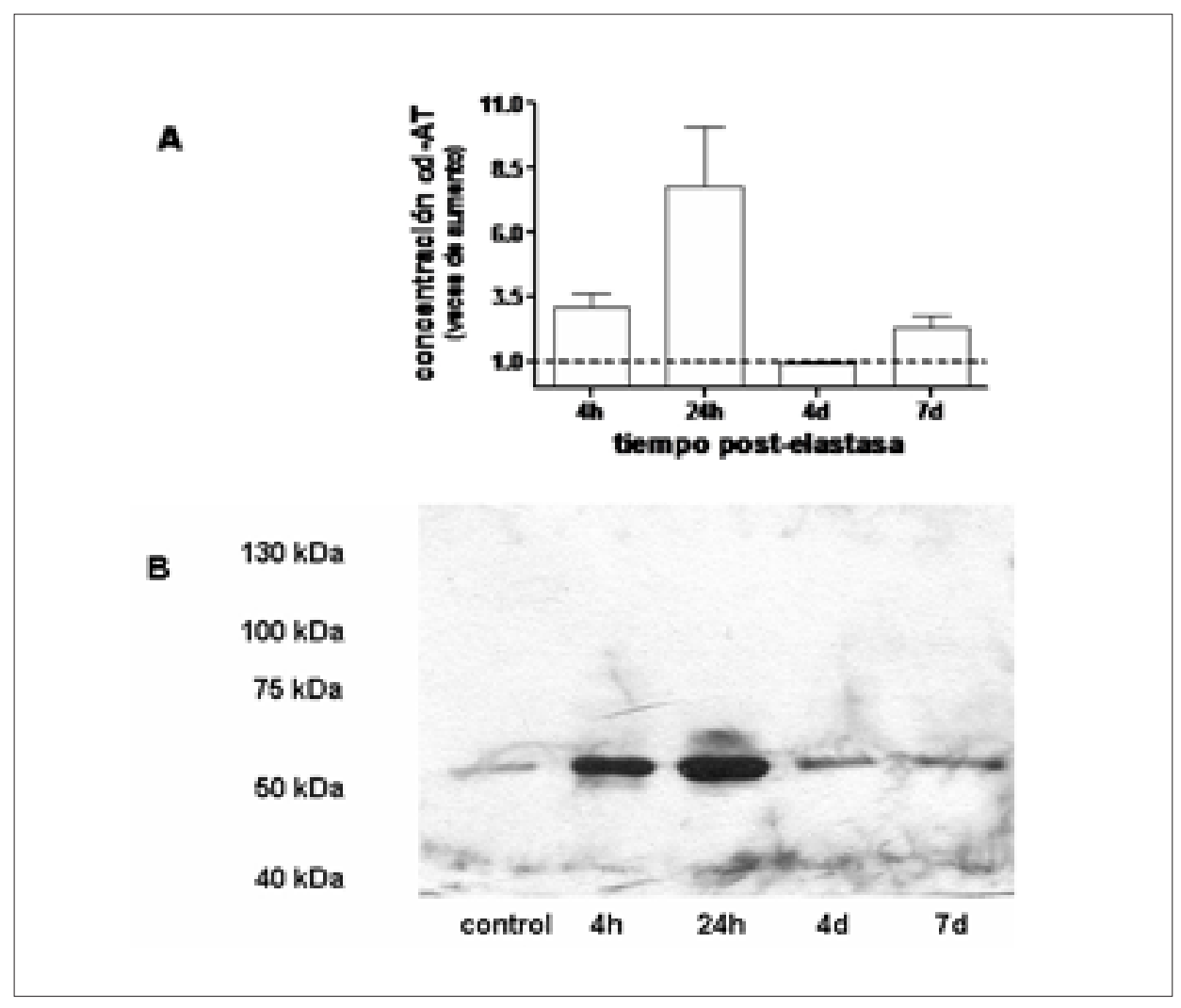

Figura 2. Análisis densitométrico en condiciones desnaturantes de los niveles de $\alpha 1-\mathrm{AT}$ en el LBA a distintos tiempos después de la instilación de elastasa. El gráfico superior (A) ilustra los niveles de inmuno-reactividad para $\alpha 1-$ AT promedio $(n=5)$ obtenidos por análisis densitométrico y expresados como veces de aumento en relación al control. Los valores corresponden al promedio \pm error estándar de los LBA obtenidos en 5 experimentos independientes mientras que la línea punteada representa el valor basal. El panel inferior (B) corresponde a un blot representativo, en condiciones desnaturantes. 
Tabla 2. Porcentajes relativos de las distintas formas inmuno-reactivas de $\alpha 1-A T$ en LBA después de la instilación de elastasa

\begin{tabular}{lccccr}
\hline Tamaño molecular & Control & 4 horas & 24 horas & 4 días & $\mathbf{7}$ días \\
\hline$<40$ kDa (\%) & 7,5 & 20,5 & 37,4 & 28,7 & 3,2 \\
$\sim 52$ kDa (\%) & 43,9 & 36,1 & 25,0 & 38,9 & 49,1 \\
$>75$ kDa (\%) & 48,6 & 29,5 & 20,0 & 32,4 & 44,7 \\
$>100$ kDa (\%) & 0,0 & 13,8 & 17,6 & 0,0 & 3,0 \\
\hline
\end{tabular}

$* *: \mathrm{p}<0,01$

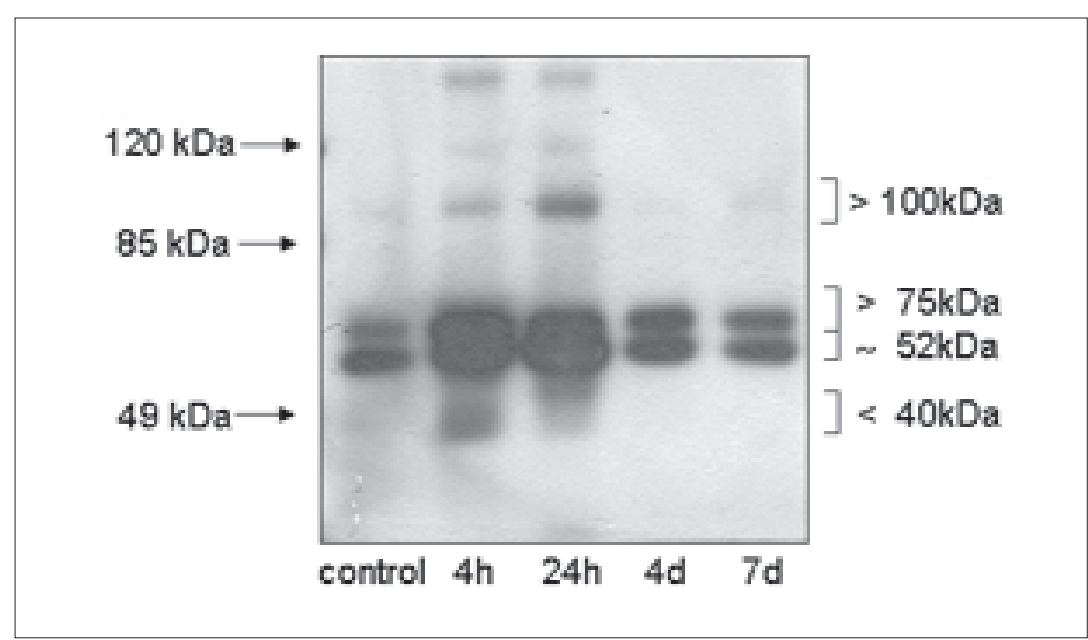

Figura 3. Fotografía de las bandas inmuno-reactivas a $\alpha 1$-AT de un western blot realizado utilizando las mismas muestras de la Figura 2, para el cual se utilizaron condiciones no desnaturantes. Se ilustra un perfil electroforético distinto. En el borde derecho de la figura están representados los tamaños moleculares de las bandas inmuno-reactivas encontradas. $\alpha 1-\mathrm{AT}$ no sólo se encuentra como una banda de $52 \mathrm{kDa}$ sino que además formando complejos y como producto de degradación.

alcanza un máximo de 7-8 veces el valor del control a las 24 horas. Este máximo coincide con el peak de aumento en proteínas totales medidas con el método de Bradford, que también es de 7 veces el valor del control (Tabla 1).

Distintas formas inmuno-reactivas de la $\alpha 1$ AT en el LBA sometido a electroforesis en condiciones no desnaturantes.

A diferencia de lo descrito para los geles en condiciones desnaturantes, cuando la electroforesis se realiza en condiciones no desnaturantes, es decir sin modificar la estructura terciaria o los complejos que forma la $a 1-\mathrm{AT}$, se identifican bandas inmuno-reactivas de diferente movilidad electroforética ${ }^{10}$. En el blot representativo mostrado en la Figura 3 es posible observar en el control, dos bandas inmuno-reactivas a $\alpha 1$ AT; una más intensa, de peso molecular $52 \mathrm{kDa}$ y otra de tamaño molecular mayor a $75 \mathrm{kDa}$, que probablemente corresponde a un complejo entre la $\alpha 1-\mathrm{AT}$ y la elastasa $(30 \mathrm{kDa})^{10,13}$.

Después de la instilación de elastasa es posible distinguir dos efectos en las primeras 24 horas: a) un significativo aumento en la intensidad de las bandas presentes en el control y b) la aparición de dos nuevas zonas inmuno-reactivas:

- Una banda de tamaño molecular inferior a 40 $\mathrm{kDa}$.

- Una banda de tamaño molecular superior a 100 kDa.

La Tabla 2 muestra el promedio de los resultados de 5 electroforesis en condiciones no desnaturantes. En ella se muestra que la participación de las distintas formas descritas en la inmuno-reactividad total para cada tiempo postelastasa es variable y las principales diferencias con el control se observan en las primeras 24 horas. 


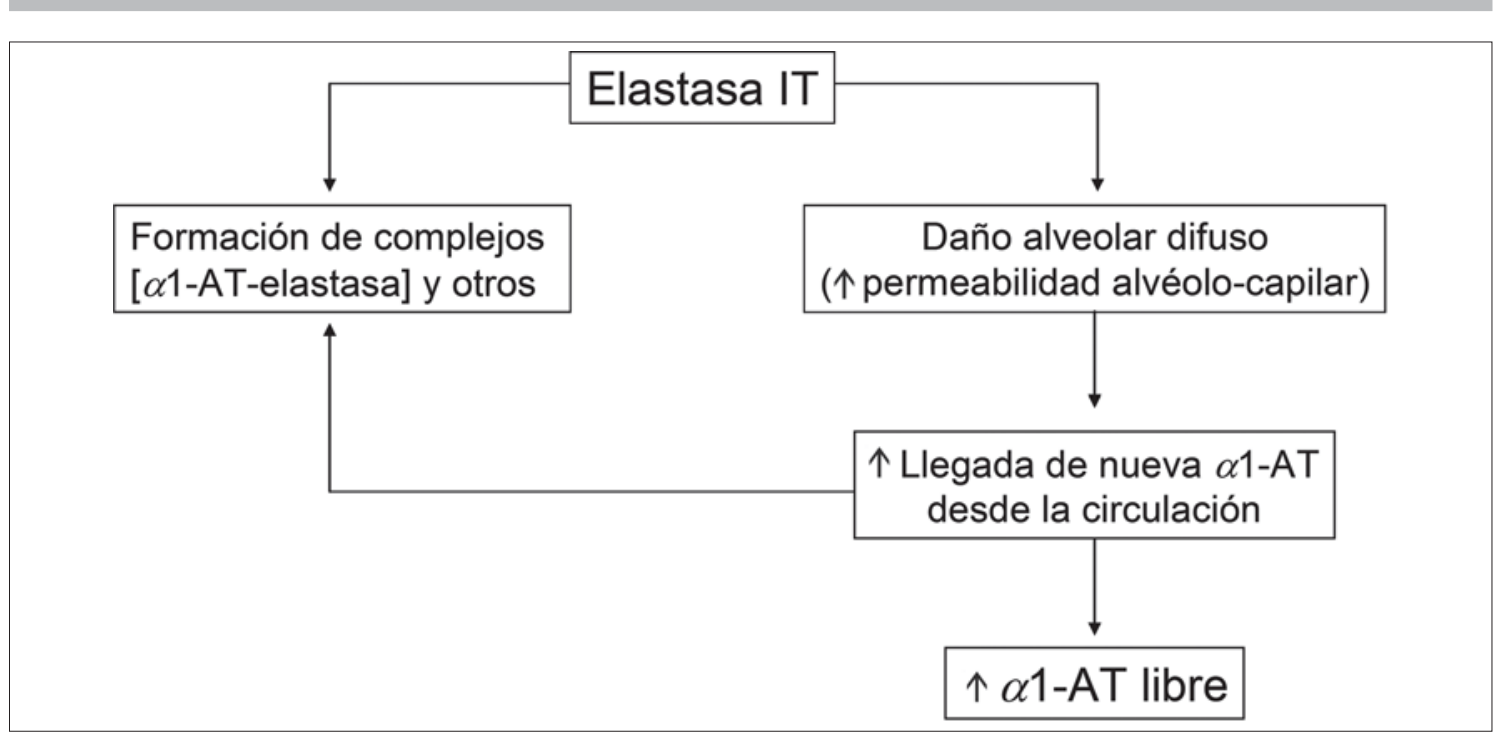

Figura 4. Secuencia de eventos que involucran a la $\alpha 1-\mathrm{AT}$ en respuesta a la instilación intratraqueal (IT) de elastasa y que contribuyen a limitar el daño pulmonar. $\uparrow=$ aumento.

Si bien en la Tabla 2 la proporción de $\alpha 1-\mathrm{AT}$ libre (banda $52 \mathrm{kDa}$ ) es de $25 \%$ a las 24 horas, valor inferior al $43,9 \%$ presente en el control, debe considerarse que de acuerdo a la Figura 2, el aumento en $\alpha 1$-AT total es de ocho veces, por lo que a pesar de encontrarse la $\alpha 1-\mathrm{AT}$ formando distintos complejos y posiblemente en parte degradada (< $40 \mathrm{kDa})$, la concentración de $\alpha 1$-AT libre (52 $\mathrm{kDa}$ ) es varias veces superior a la del control.

\section{Discusión}

Los resultados de este estudio muestran que mediante técnicas electroforéticas y de inmunodetección por western blot, es posible identificar distintas formas de $\alpha 1$-AT en los espacios aéreos del pulmón, las que varían dependiendo del tiempo de evolución del daño inducido por la instilación de elastasa.

Además de la banda de $52 \mathrm{kDa}$ correspondiente a la $\alpha 1$-AT nativa ${ }^{14}$, encontramos evidencias de la participación de esta antiproteasa en la formación de complejos de distintos tamaños. El complejo con mayor probabilidad de formarse es el de la $\alpha 1-A T$ con la elastasa instilada. La banda de peso molecular > $75 \mathrm{kDa}$ correspondería a este complejo ${ }^{10-17}$. Otras proteínas con las que es capaz de reaccionar la $\alpha 1$-AT incluyen: a) proteínas propias del LBA como por ejemplo urokinasa ${ }^{20}$; y b) proteínas que aparecerían en éste, debido al aumento de permeabilidad alvéolo-capilar ${ }^{18-21}$. Por último, se ha des- crito que la $\alpha 1$-AT puede formar complejos consigo misma (banda $>100 \mathrm{kDa}^{3,10}$. De igual forma identificamos bandas inmuno-reactivas de menor tamaño, sugerentes de fenómenos de proteólisis que afectarían a la $\alpha 1-\mathrm{AT}^{23-24}$.

La instilación IT de elastasa en distintos animales de experimentación produce un daño alveolar difuso caracterizado por el aumento en la permeabilidad alvéolo-capilar con edema, hemorragia e infiltración de células inflamatorias, el que tiene su máxima intensidad a las 24 horas $^{25}$. En estas condiciones se espera que la $\alpha 1-$ AT experimente diversas modificaciones tendientes a contrarrestar el daño proteolítico disminuyendo su actividad. Entre ellas están: la formación de complejos con la elastasa instilada ${ }^{11}$, la oxidación de su sitio activo por oxidantes liberados desde las células inflamatorias reclutadas al pulmón ${ }^{26-30}$, y la inactivación proteolítica como fue descrita por Banda y cols s3,24. $^{23}$.

Sin embargo, en un estudio previo en nuestro laboratorio encontramos que el LBA de la rata después de la instilación IT de elastasa presenta un aumento en la actividad de $\alpha 1-\mathrm{AT}^{8}$, sugiriendo la existencia de algún mecanismo que contribuye a aumentar la concentración de $\alpha 1$-AT activa en los espacios aéreos.

El estudio actual muestra (Figura 4) que la concentración de $\alpha 1$-AT aumenta en el LBA unas 7-8 veces, gracias a la llegada masiva de proteínas séricas debido al aumento de la permeabilidad alvéolo-capilar (Figura 1), que es parte del daño alveolar difuso inducido por la elastasa instilada ${ }^{25}$. 
El aumento de permeabilidad es un fenómeno conocido y es la base del daño alveolar difuso producido por distintos agentes. El curso temporal del aumento de permeabilidad en nuestro trabajo concuerda con los resultados publicados por Schmid y cols ${ }^{31}$ quienes encontraron que la permeabilidad alvéolo-capilar en el hámster, medida con marcadores radioactivos aumenta desde las primeras horas post elastasa, permanece aumentada a los tres días y se normaliza al quinto día

El curso temporal del aumento en la concentración de $\alpha 1$-AT coincide con el curso temporal del aumento en la actividad de la $\alpha 1-A T$ mostrada por Salazar y cols, si bien las magnitudes respectivas no son las mismas.

De acuerdo a los resultados obtenidos de la electroforesis en condiciones no desnaturantes (Figura 3), a las 24 h post-elastasa, más del $74 \%$ de la $\alpha 1-\mathrm{AT}$ se encuentra formando complejos de distinto tamaño o como fragmento proteolítico. Sólo el $25 \%$ se encuentra como una banda de $52 \mathrm{kDa}$, parte de la cual es responsable del aumento del $60 \%$ en la actividad de $\alpha 1$-AT descrito por Salazar y cols ${ }^{8}$. Estos resultados sugieren que en el modelo de instilación IT de elastasa en la rata, los fenómenos de oxidación que inactivarían a la $\alpha 1$-AT y que han sido descritos principalmente en relación al daño por humo de cigarrillo ${ }^{14-18}$, son de menor relevancia que los fenómenos de formación de complejos.

En conclusión, la llegada masiva de $\alpha 1-A T$ desde la circulación provee de defensa más que suficiente para limitar el daño inducido por la elastasa en la rata. Fenómenos como los descritos pueden participar en otros procesos inflamatorios pulmonares. Se desconoce si los fenómenos encontrados en la rata se presentan también en el hámster, una especie que se caracteriza por ser deficiente en $\alpha 1$-AT y por su mayor vulnerabilidad al daño pulmonar inducido por elastasa $^{32}$. Es posible que diferencias interespecies en la respuesta de la $\alpha 1-\mathrm{AT}$ en los espacios aéreos post-elastasa se relacionen con diferencias inter-especies en la magnitud del enfisema resultante $\mathrm{e}^{32,33}$.

\section{Bibliografía}

1.- GADEK J E, FELLS G A, ZIMMERMAN R L, RENNARD S I, CRYSTAL R G. Antielastases of the human alveolar structures. Implications for the protease-antiprotease theory of emphysema. J Clin Invest 1981; 68: 889-98.

2.- CRYSTAL R G. Alpha-1-antitrypsin deficiency, emphysema, and liver disease. Genetic basis and strategies for therapy. J Clin Invest 1990; 85: 134352.

3.- LOMAS D A, MAHADEVA R. Alpha-1-antitrypsin polymerization and the serpinopathies: pathobiology and prospects for therapy. J Clin Invest 2002; 110: 1585-90.

4.- VOGELMEIER C, HUBBARD R C, FELLS G A, SCHNEBLI H P, THOMPSON R C, FRITZ H, et al. Anti-neutrophil elastase defense of the normal human respiratory epithelial surface provided by the secretory leukoprotease inhibitor. J Clin Invest 1991; 87: 4828.

5.- BIETH JG. In vivo significance of kinetic constants of protein proteinase inhibitors. Biochem Med 1984; 32: 387 - 97

6.- YANG P, BAMLET $W$ R, SUN Z, EBBERT J O, AUBRY M C, TAYLOR W R, et al. Alpha1-antitrypsin and neutrophil elastase imbalance and lung cancer risk. Chest 2005; 128: 445-52. Erratum in: Chest 2006; 129 (1): 216. Krowka, Michael

7.- GROSS P, PFITZER E A, TOLKER E, BABYAK M A, KASCHAK M. Experimental emphysema: its production with papain in normal and silicotic rats. Arch Environ Health 1965; 11: 50-8.

8.- SALAZAR I, RAMÍREZ A, LIBERONA L, SÁEZ C, BORZONE G. Actividad antielastasa del lavado broncoalveolar en ratas y hámsters postinstilación intratraqueal de elastasa. Rev Chil Enf Respir 2005; 21: 284 (Resumen).

9.- BRADFORD M M. A rapid and sensitive method for quantitation of microgram quantities of protein utilizing the principle of protein dye binding. Anal Biochem 1976; 72: 248-54.

10.- BAUMSTARK J S. Quantitative immunofixation of proteins following zone electrophoresis in agarose gel: application to the determination of the stoichiometry of the alpha1-antitrypsin-elastase interaction. J Immunol Methods 1978; 23: 79-89.

11.- KNOELL D L, RALSTON D R, COULTER K R, WEWERS M D. Alpha1-antitrypsin and protease complexation is induced by lipopolysaccharide, interleukin-1beta, and tumor necrosis factor-alpha in monocytes. Am J Respir Crit Care Med 1998; 157: 246-55.

12.- TUKEY J W. Some selected quick and easy methods of statistical analysis. Trans NY Acad Sci 1953; 16: 88-97.

13.- KANG U B, BAEK J H, RYU S H, KIM J, YU M H, LEE C. Kinetic mechanism of protease inhibition by alpha1-antitrypsin. Biochem Biophys Res Commun 2004; 323: 409-15.

14.- TRAVIS J, SALVESEN G S. Human plasma proteinase inhibitors. Annu Rev Biochem 1983; 52: 655-709.

15.- BAUMSTARK J S. A zone electrophoretic-isoelectric focusing study on the inactivation of human alpha1antitrypsin by elastase. Appl Theor Electrophor 1988; 1: 65-71.

16.- BEATTY K, BIETH J, TRAVIS J. Kinetics of association of serine proteinases with native and oxidized alpha-1-proteinase inhibitor and alpha-1antichymotrypsin. J Biol Chem 1980; 255: 3931-4.

17.- GREENE C, TAGGART C, LOWE G, GALLAGHER P, McELVANEY N, O'NEILL S. Local impairment of anti-neutrophil elastase capacity in communityacquired pneumonia J Infect Dis 2003; 188: 769-76.

18.- BLOOM J W, HUNTER M J. Interactions of alpha1antitrypsin with trypsin and chymotrypsin. J Biol 
Chem 1978; 253: 547-59.

19.- MOROI M, YAMASAKI M, AOKI N. Association of human alpha1-antitrypsin with anhydrotrypsin. J Biochem (Tokio) 1975; 78:925-8.

20.- CLEMMENSEN I, CHRISTENSEN F. Inhibition of urokinase by complex formation with human alpha1antitrypsin. Biochim Biophys Acta 1976; 429: 591-9.

21.- SCOTT L J, EVANS E L, DAWES P T, RUSSELL G I, MATTEY D L. Comparison of IgA-alpha1-antitrypsin levels in rheumatoid arthritis and seronegative oligoarthritis: complex formation is not associated with inflammation per se. Br. J. Rheumatol 1998; 37: 398-404.

22.- MULGREW A T, TAGGART C C, LAWLESS M W, GREENE C M, BRANTLY M L, O’NEILL S J, et al. $\mathrm{Z}$ alpha1-antitrypsin polymerizes in the lung and acts as a neutrophil chemoattractant. Chest 2004; 125: 1952-7.

23.- BANDA M J, CLARK E J, WERB Z. Regulation of alpha1-proteinase inhibitor function by rabbit alveolar macrophages. Evidence for proteolytic rather than oxidative inactivation. J Clin Invest 1985; 75: 175862.

24.- HERCZ A. Proteolytic cleavages in alpha1-antitrypsin and micro-heterogeneity. Biochem Biophys Res Commun 1985; 128: 199-203.

25.- LUCEY E C, GOLDSTEIN R H, STONE P J, SNIDER G L. Remodeling of alveolar walls after elastase treatment of hamsters: Results of elastin and collagen mRNA in situ hybridization. Am J Respir Crit Care Med 1998; 158: 555-64.

26.- CARP H, JANOFF A. In vitro suppression of serum elastase-inhibitory capacity by reactive oxygen species generated by phagocytosing polymorphonuclear leukocytes. J Clin Invest 1979; 63: 793-7.

27.- CARP H, JANOFF A. Potential mediator of inflammation. Phagocyte-derived oxidants suppress the elastase-inhibitory capacity of alpha1-proteinase inhibitor in vitro. J Clin Invest 1980; 66: 987-95.

28.- JANOFF A, CARP H, LEE D K, DREW R T. Cigarette smoke inhalation decreases alpha1-antitrypsin activity in rat lung. Science 1979; 206: 1313-4.

29.- CLARK R A, STONE P J, EL HAG A, CALORE J D, FRANZBLAU C. Myeloperoxidase-catalyzed inactivation of alpha1-protease inhibitor by human neutrophils. J Biol Chem 1981; 256: 3348-53.

30.- McELVANEY N, CRYSTAL R. Antiproteases and lung defense. The Lung Scientific Foundations. Segunda Edición. Editado por R Crystal, J West y cols. LippincotRaven Publisher, Filadelfia, USA. 1997 págs: 221935.

31.- SCHMID R P, WANGENSTEEN D, HOIDAL J, GOSNELL B, NIEWOEHNER D. Effects of elastase and cigarettes smoke on alveolar epithelial permeability. J Appl Physiol 1985; 59: 96-100.

32.- BORZONE G, LIBERONA L, OLMOS P, SÁEZ C, MENESES M, REYES T, et al. Rat and hamster species differences in susceptibility to elastase-induced pulmonary emphysema relate to differences in elastase inhibitory capacity. Am J Physiol Regul Integ Comp Physiol 2007; 293: R1342-9.

33.- BORZONE G, RAMÍREZ B, REYES T, MORENO R, LISBOA C, GONZÁLEZ S. Experimental pulmonary emphysema in the rats. Inflammatory phenomena and progression of lung damage. Rev Méd Chil 1998; 126: 1153-60.
Correspondencia a:

Andrea Vecchiola Cárdenas

Departamento de Enfermedades Respiratorias y

Centro de Investigaciones Médicas.

Facultad de Medicina.

Pontificia Universidad Católica de Chile.

Marcoleta 391, Piso 3.

Teléfono: 354-3815; Fax: 633-5255

E-mail avecchi@med.puc.cl 